\title{
Analysis For Flow Field Characteristics of Purified Gas Flue Based on ANSYS
}

$$
\text { Haiming Zheng }{ }^{1, a^{*}} \text {, Jingjun Yang }{ }^{2, b}
$$

\author{
${ }^{1}$ School of Mechanical Engineering North China Electric Power University, Baoding Hebei China \\ ${ }^{2}$ School of Mechanical Engineering North China Electric Power University, Baoding Hebei China \\ a haimingzheng@163.com, b15933912536@163.com
}

\begin{abstract}
Keyword: Purified gas flue; Flow field characteristics; Flow measuring point
Abstract. Regarding issue of poor measurement accuracy on gas flow in purified gas flue after processed by coal-fired power plant desulfurization system, we make numerical simulation through Workbench of ANSYS towards flow properties within purified gas flue of No.8 unit Yangcheng power plant processed by gas desulfurization system, analyze different flow field distribution under different gas flow. The result show that change of the gas flow has small influence on velocity distribution inside the flue, the most symmetrical area appears at central area of lower level flue, after three times diversion the velocity distribution in upper level flue at vertical and horizontal direction are not balance, therefore, central area of lower level flue will be the best flow measuring point.
\end{abstract}

\section{Introduction}

Flue gas flow discharge at heat-engine plant is a vital data for environmental monitor and most of online flow meters are applied on flue in front of chimney [1,2]. After desulfurization project due to space limitation, flue connecting purified gas and chimney is often short and with $90^{\circ}$ turning, thus, it brings trouble for measuring point application as flow field disturbance existed during turning area. Inappropriate application point may lead to large fluctuation and low accuracy on flow value monitored even without value for long time, which cannot meet requirements of environmental monitor [3]. By using ANSYS and flow distribution in purified gas flue after desulfurization, we get the conclusion that central area of lower level flue will be the best point for flow measuring.

\section{Calculation model and boundary conditions}

\section{Rectangular flue calculation model}

Geometric model for calculation adopts actual purified gas flue agency data after desulfurization of No.8 unit Yangcheng power plant, computational domain begins with export joint of gas-gas heater (GGH) to joint part of chimney flue and purified gas flue, geometric model is drawn by Proe as picture 1. According to picture1, gas flows to down flue from GGH, arrive at lower level flue after turning, then goes up to upper level flue after further turning finally reach joint part of chimney flue.

Calculation model adopts actual structure size, coordinate axis goes as follows: $\mathrm{X}$ axis is the mainstream direction of flue gas, zero setting places at the axis of the GGH export flue, Y axis is height direction, $\mathrm{Z}$ axis is depth of the flue. Besides, the lower flue includes down flue after GGH, turning, lower level flue and updraught flue after turning; upper horizontal bending flue mainly refer to the horizontal bending flue connecting from updraught flue after turning and fit for chimney position. 


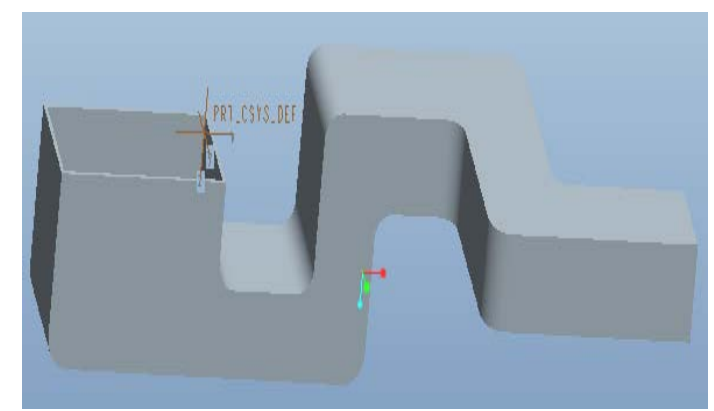

Picture 1 Purified gas flue model diagram

With effects of airflows in front of main gas flue, the flowing condition of airflows up to monitoring section is uneven, and the monitored flow value from flow instrument presents a great fluctuation and low precision. Therefore, guide plate shall be set up in rectangle gas flue to adjust the air flow distribution within the gas flue [4].

\section{Boundary conditions}

On a basis of combination of numerical simulation and experimental data, boundary conditions have been set as below:

(1)Input adopts the boundary condition of flow to get airflow even and vertical. Two groups of data have been analyzed. The flow of first group is $662.1 \mathrm{Nm} 3 / \mathrm{s}$, and the second group is $360 \mathrm{Nm} 3 / \mathrm{s}$. Flue-gas temperature is calculated as the design value $80^{\circ} \mathrm{C}$.

(2)The outlet adopts the boundary condition of pressure. The pressure value of the first group is 432 $\mathrm{Pa}$, and the second group is $230 \mathrm{~Pa}$.

(3)To simplify calculation, flue gas is deemed as pure gas without regard to the effects of different flue gas components on calculation results. No slip is set between flue gas flow and wall surface, and the wall surface will be treated upon insulation[5].

\section{Numerical calculation result analysis}

\section{Flow field characteristics analysis inside purified gas flue}

The flow field distribution diagram of purified flue gas under flue gas flow of the first group is shown in Picture 2 and 3. Therein, Picture 2 is flow field velocity vector distribution diagram inside purified gas flue, while Picture 3 is flue gas velocity flow line distribution diagram inside purified flue gas.

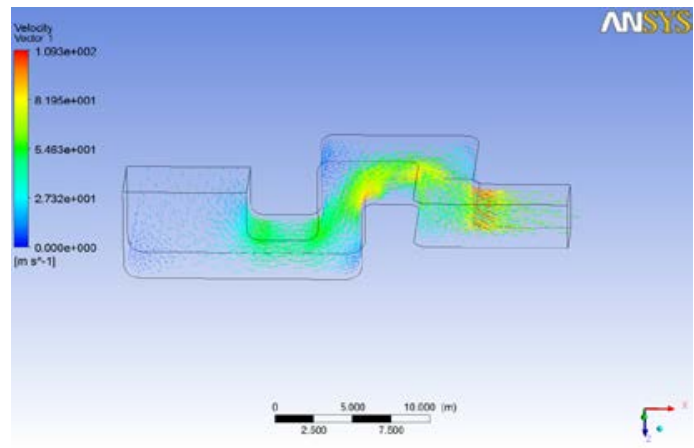

Picture 2 Flow field velocity vector distribution diagram inside purified gas flue 


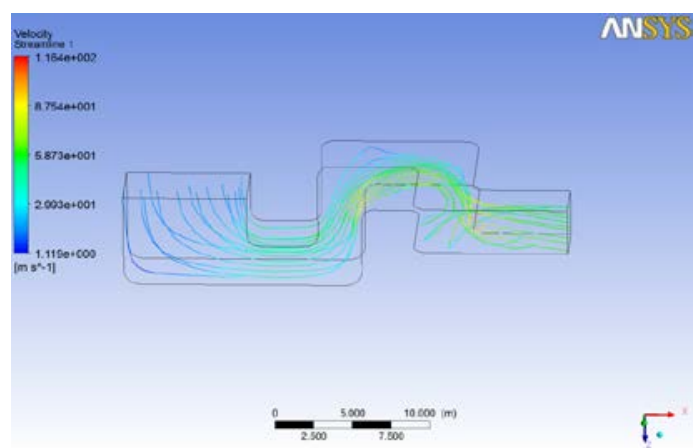

Picture 3 Flue gas velocity flow line distribution diagram inside purified gas flue

From Picture 2 and 3, velocity field distribution inside gas flue: flue gas makes a turn from the gas flue after GGH to access to horizontal flue of bottom, and the velocity of flue gas tends to be even. The flue gas continues to flow from level to vertical turn. Except low-speed areas exist at turn points in upstream process, the velocity is also relatively even; but the flue gas turns to horizontal mobility again, and the existence of verticality-to-level turn and flue horizontal turn set for adapting to the chimney leads to a upside turning area before horizontal-turn flue and a low-velocity backflow eddy area; afterwards, the short size of two continuous turns and upside horizontal bending gas flue leads to a heavy asymmetry of velocity distribution within the whole upside horizontal bending gas flue.

It is concluded that the distribution of velocity is even in the down part of the flue by analyzing distribution of flow field of group 1, and the most even zone is the middle of the horizontal flue and velocity does not vary much; The distribution of velocity in the upper part is seriously uneven in vertical section after 3 turns; Low velocity or high velocity appear partly in the arc of each turn in the flue.

\section{Effect on the distribution of velocity in the flue by different exhaust gas flow}

In order to show effect on the distribution of flow field in the flue by different exhaust gas flow, we make numerical simulations under 2 different exhaust gas flows. Picture 4 is showing the velocity vector distribution in the flow field of Group 2 exhausted gas, and Picture 5 is showing the velocity distribution of purified exhausted gas in the flue.

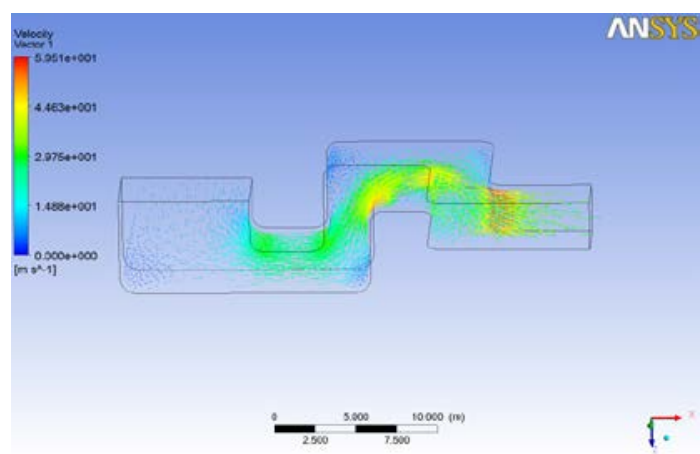

Picture 4 The velocity distribution of the flow field in the purified exhausted gas flue

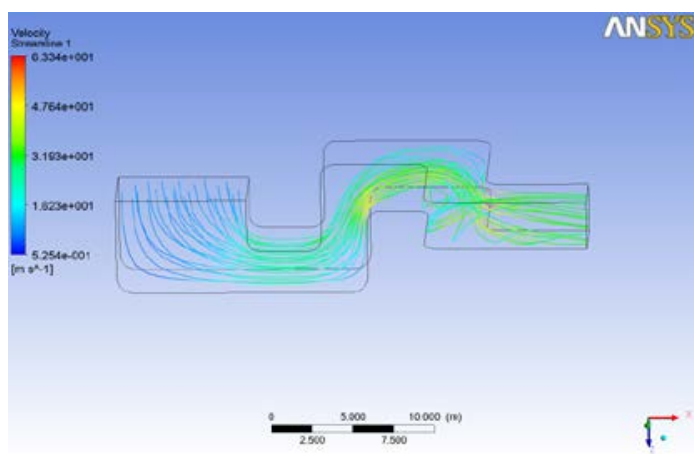

Picture 5 The velocity distribution of purified exhausted gas in the flue. 
Contrasting the two Pictures of numerical simulations, we know that the velocity distribution in the flue is almost the same but the velocity value and the variation of exhaust gas flow does not affect very much the velocity distribution in the flue.

\section{Summary}

(1) The distribution of velocity is even in the down part of the flue, and the most even zone is the middle of the horizontal flue; The distribution of velocity in the upper part is seriously uneven both vertically and horizontally after 3 turns.

(2) The variation of exhaust gas flow does not affect very much the velocity distribution in the flue. By contrasting the 2 numerical simulations of different exhausted gas flow, we know that the velocity distribution in the flue is almost the same but the velocity value.

(3) By analyzing the data of flow field in the purified exhausted gas flue of No.8 generator set in Yangcheng Power Plant, we find that the flow is the most even in the horizontal middle of the down part of the flue. So, it is preferable to install the flow detector here.

(4)The flow detecting spot is reasonably chosen by calculation of 2 numerical simulations, thus the precision of flow detection is improved.

\section{Acknowledgment}

This work is supported by National Natural Science Foundation of China (11174078), Hebei Provincial Natural Science Foundation of China (E2012502046), Fundamental Research Funds for the Central Universities (12MS102).

\section{References}

[1] Zhang Zhenyi, Zou Lei. Power Plant net gas flue flow field characteristics analysis and flow measurement point selection[J]. Jiangsu motor engineering, 2014,33 (4): 75-77.DOI:10.3969/j.issn.1009-0665.2014.04.021.

[2] Zou Lei, Yue Junfeng, Ning Xinyu, Liang Shao Hua, Zhang Enxian, Huang Lei. Based on CFD calculation of net of flue gas in gas flow measurement point selection [A]. The seventh power industry energy saving emission reduction seminar the set

[3] Wang Wanlin, Qi Xiaojuan. Research on the measurement of flue gas discharge of power plant [J]. Zhejiang power, 19 (6):17 - 2009

[4] Wang Wanlin, Qi Xiaojuan, Li Fengrui. Improved CFD simulation of gas flow distribution in the flue gas of power plant after desulfurization [J]. of East China power, 2013,41 (1): 220-223.

[5] Wang Fujun. Computational fluid dynamics analysis CFD software principle and application [M]. Beijing: Tsinghua University press.2004:7-11. 